\title{
Thermal effects on a global monopole with Robin boundary conditions
}

\author{
Lissa de Souza Campos $\oplus^{1,2, *}$ and João Paulo M. Pitelli@ ${ }^{3, \uparrow}$ \\ ${ }^{1}$ Dipartimento di Fisica, Università degli Studi di Pavia, Via Bassi, 6, 27100 Pavia, Italy \\ ${ }^{2}$ Istituto Nazionale di Fisica Nucleare, Sezione di Pavia, Via Bassi, 6, 27100 Pavia, Italy \\ ${ }^{3}$ Departamento de Matemática Aplicada, Universidade Estadual de Campinas, \\ 13083-859 Campinas, São Paulo, Brazil
}

(Received 1 September 2021; accepted 1 October 2021; published 21 October 2021)

\begin{abstract}
Within quantum field theory on a global monopole spacetime, we study thermal effects on a naked singularity and their relation to boundary conditions. We first obtain the two-point functions for the ground state and for thermal states of a massive, arbitrarily coupled, free scalar field compatible with Robin boundary conditions at the singularity. We then probe these states using a static Unruh-Dewitt particle detector. The transition rate is analyzed for the particular cases of massless minimally or conformally coupled fields at finite temperature. To interpret the detector's behavior, we compute the thermal contribution to the ground-state fluctuations and to the energy density. We verify that the behavior of the transition rate, the fluctuations, and the energy density are closely intertwined. In addition, we find that these renormalized quantities remain finite at the singularity for, and only for, the Dirichlet boundary condition.
\end{abstract}

DOI: 10.1103/PhysRevD.104.085020

\section{INTRODUCTION}

The singularity theorems from the 1960s and 1970s clarified that singularities are not just a consequence of highly symmetrical scenarios - they are endemic to general relativity [1]. Notwithstanding, it is not unreasonable to expect naked singularities to be absent in nature. On the one hand, the cosmic censorship conjecture stipulates that singularities, in our Universe, shall always be hidden by a horizon. On the other hand, naked singularities naturally emerge in some (theoretical) scenarios-white holes, cosmic strings, and global monopoles [2]. Ultimately, the consensus is probably that the matter can only be fully resolved within a theory of quantum gravity. Be that as it may, semiclassical analysis such as quantum field theory on curved spacetimes can elucidate the path towards a better understanding of the interface between quantum physics and general relativity.

Semiclassical analyses have brought to light several physical phenomena. Amongst these, two are noteworthy: Hawking radiation, which concerns the particle production constituting the final process in the life of a black hole [3], and the quantum dressing of a naked singularity due to

\footnotetext{
"lissa.desouzacampos01@universitadipavia.it †pitelli@unicamp.br
}

Published by the American Physical Society under the terms of the Creative Commons Attribution 4.0 International license. Further distribution of this work must maintain attribution to the author(s) and the published article's title, journal citation, and DOI. Funded by SCOAP. backreaction effects. Regarding the latter, it has been shown that scalar perturbations of a negative mass Bañados-Teitelboim-Zanelli black hole brings about a horizon of Planckian radius covering its previously naked singularity [4]. Maybe nature indeed hinders naked singularities; however, there might be more than one mechanism to do so besides the existence of a horizon. With Hawking radiation in mind, one may wonder if evaporation could be another one.

Motivated by the above discussion, in this work we study thermal effects within quantum field theory on a naked singularity spacetime and their dependence on the admissible boundary conditions. Specifically, we consider a free, scalar quantum field theory on a global monopole spacetime. Global monopoles arise when a global symmetry is spontaneously broken [2]. According to grand unified theories, they can result from phase transitions in the early Universe. A global monopole spacetime $\mathcal{M}$ is described by the line element

$$
d s^{2}=-d t^{2}+d r^{2}+\alpha^{2} r^{2} d \theta^{2}+\alpha^{2} r^{2} \sin ^{2} \theta d \varphi^{2},
$$

where $t \in \mathbb{R}$ yields a global, timelike, irrotational Killing vector field $\partial_{t}, r \in(0, \infty), \theta \in[0, \pi)$, and $\varphi \in[0,2 \pi)$. The parameter $\alpha \in(0,1)$ gives rise to a solid angular deficit. In particular, the hypersurface $\theta=\frac{\pi}{2}$ corresponds to a cone with a deficit angle of $2 \pi(1-\alpha)$. In these coordinates, the Ricci and the Kretschmann scalars are, respectively,

$$
\mathbf{R}=\frac{2\left(1-\alpha^{2}\right)}{\alpha^{2} r^{2}} \quad \text { and } \quad \mathbf{K}=\mathbf{R}^{2}
$$


and the metric associated to (1) solves Einstein field equations with a classical energy-momentum tensor whose only nonvanishing components are

$$
T_{t t}=-T_{r r}=\frac{\mathbf{R}}{2} .
$$

It follows that the singularity at $r \rightarrow 0$ is naked, timelike, and of curvature type. Accordingly, $\mathcal{M}$ is a static, geodesically incomplete, nonglobally hyperbolic spacetime on which the propagation of quantum fields depends on the choice of boundary condition at the singularity.

For the establishment of a quantum field theoretical framework, the first step consists of obtaining physically sensible two-point functions. Since global monopoles are not globally hyperbolic spacetimes, the Klein-Gordon equation gives rise to an initial-boundary value problem. That is, given suitable initial data on a spacelike surface, corresponding solutions, if they exist, are specified by boundary conditions, yielding inequivalent dynamics. Amongst the possible boundary conditions to be imposed, we restrict our attention to the ones that generate physically sensible dynamics in the sense of Ishibashi and Wald $[5,6]$. Namely, we focus on the ones that bring about self-adjoint extensions of the radial part of the KleinGordon operator.

In Ref. [7], it was shown that the sensible dynamics for a scalar field around a global monopole are prescribed by Robin boundary conditions at the singularity. Given this infinite class of nonequivalent dynamics, Ref. [8] extended the analysis of Dirichlet quantum fields in the global monopole spacetime considered in Ref. [9] to include these nontrivial Robin boundary conditions. Following the same reasoning, we study thermal states for quantum fields with Robin boundary conditions, generalizing the results of Ref. [10], where only the Dirichlet boundary condition was considered.

Our interest lies not only in investigating thermal effects on naked singularities, but also in studying them together with the impact of having different, inequivalent, physically sensible dynamics engendered by the naked singularity. With the corresponding two-point functions in hand, we can probe these different quantum states within the particle detector approach. To this goal, we consider an UnruhDeWitt detector following a static trajectory and interacting with a quantum state on a global monopole spacetime via a monopole-type Hamiltonian operator; see, e.g., Ref. [11] and the references therein. In the infinite interaction time limit and up to first-order perturbation theory, the instantaneous transition rate $\dot{\mathcal{F}}$ of the detector coincides with the Fourier transform of the pullback $\mathcal{G}(s)$ along the detector trajectory, parametrized by the proper time interval $s=\tau-\tau^{\prime}$, of the two-point function of the underlying field evaluated at the detector's energy gap $\Omega$ :

$$
\dot{\mathcal{F}}=\int_{\mathbb{R}} d s e^{-i \Omega s} \mathcal{G}(s) .
$$

The transition rate characterizes the probabilities of excitations, for $\Omega>0$, and deexcitations, for $\Omega<0$, of the detector. In general, the transition rate can be seen as a function of the detector's trajectory, of its energy gap, and, of course, of the quantum state to which it is coupled. We obtain expressions that can easily be studied numerically for an arbitrary parameter set, but we focus on the response of a detector coupled to thermal states of massless, minimally or conformally coupled fields. In addition, for a given state on a fixed global monopole background, we see $\dot{\mathcal{F}}$ merely as a function of the distance between the detector and the naked singularity by fixing an arbitrary energy gap $\Omega>0$, and we compare its behavior for different deficit angles and different boundary conditions.

Last, to paint a better picture of the consequences of taking thermal effects together with different boundary conditions into account, we compute the thermal contributions to the expectation value of the field squared and the energy density of renormalized thermal states. Such quantities have been computed at the ground state with Dirichlet and Robin boundary conditions [8,9], and at thermal states with the Dirichlet boundary condition [10]. Yet, as for the two-point functions, considering both thermality and general boundary conditions renders a novelty character to our work.

We proceed as follows. In Sec. II, we construct the twopoint functions for the ground state and for thermal states. Then, we obtain an analytic expression for the transition rate, in Sec. III, and we study it, numerically (by truncating an infinite sum), for the case of massless, minimally and conformally coupled fields at finite temperature. In Sec. IV, we summarize the results concerning the thermal fluctuations and the energy density of the renormalized thermal state and we discuss its relation with the behavior of the detector. Final remarks are included in Sec. V.

\section{TWO-POINT FUNCTIONS}

In this section, we obtain two-point functions for the ground state and for thermal states of a free, scalar, massive quantum field theory on a global monopole spacetime $\mathcal{M}$. We avail of a standard procedure that applies on static spacetimes, as performed and detailed in Refs. [12-15]. First, in Sec. II A, we obtain the solutions of the KleinGordon equation by mode expansion. Second, we invoke spectral theory of second-order partial differential operators to study the radial equation. Its Green's function is unique up to the choice of a boundary condition at the naked singularity, as specified in Sec. II B. The symmetries of the spacetime, together with the Klein-Gordon equation, the canonical commutation relations, and the restriction to sensible dynamics completely determine the integral kernel of two-point functions for physically sensible, quasifree 
ground and thermal states of a local Hadamard form. This is detailed in Sec. II C, where we also write down their explicit expressions.

\section{A. The Klein-Gordon equation}

Let us consider a free scalar field $\Psi: \mathcal{M} \rightarrow \mathbb{R}$ with mass $m_{0} \geq 0$, coupled to the scalar curvature by a coupling parameter $\xi \geq 0$. Its dynamics is described by the KleinGordon equation:

$$
P \Psi=\left(\square-m_{0}^{2}-\xi \mathbf{R}\right) \Psi=0 .
$$

We consider solutions that can be written in the form

$$
\Psi_{\omega, \ell}(t, r, \theta, \varphi)=e^{-i \omega t} R(r) Y_{\ell}^{m}(\theta, \varphi),
$$

where $Y_{\ell}^{m}(\theta, \varphi)$ are the spherical harmonics with eigenvalues $-\ell(\ell+1)$, while the function $R(r)$ satisfies the Bessel equation

$$
R^{\prime \prime}(r)+\frac{2}{r} R^{\prime}(r)+\left(p^{2}-\frac{\lambda_{\ell, \xi, \alpha}}{r^{2}}\right) R(r)=0,
$$

with

$$
\begin{gathered}
p^{2}:=\omega^{2}-m_{0}^{2}, \\
\lambda_{\ell, \xi, \alpha}:=\frac{\ell(\ell+1)+2 \xi\left(1-\alpha^{2}\right)}{\alpha^{2}} .
\end{gathered}
$$

A basis of solutions $\left\{R_{1}, R_{2}\right\}$ of (7) is given in terms of the spherical Bessel functions of the first and second kinds ([16], Chap. 10), respectively, $j_{\nu}$ and $y_{\nu}$ :

$$
R_{1}(p r)=j_{\nu}(p r), \quad R_{2}(p r)=p y_{\nu}(p r),
$$

with index

$$
\nu:=\frac{-1+\sqrt{1+4 \lambda_{\ell, \xi, \alpha}}}{2} \geq 0 .
$$

The solutions (10) are normalized in order to have a unit Wronskian, and their dependence on $p$ is made explicit for notational convenience. Clearly, any linear combination of (10) solves the radial equation (7). However, not all of them yield self-adjoint extensions for the radial part of the Klein-Gordon operator. In the next section, we show how to restrict the space of radial solutions to a self-adjoint domain.

\section{B. Robin boundary conditions}

Self-adjoint extensions of a partial differential operator are in correspondence with its square-integrable solutions. Taking the radial equation as a Sturm-Liouville problem with eigenvalue $p^{2}$ singles out the appropriate Hilbert space of solutions - the space of square-integrable functions with respect to the measure $q(r)=r^{2}$. By direct inspection, and taking into account Weyl's endpoint classification, we find that $r \rightarrow \infty$ is a limit point, and that $r \rightarrow 0$ is a limit point for $\ell>0$, but a limit circle for $\ell=0$. By ([17], Theorem 10.4.5), it follows that there is a one-parameter family of (generalized) Robin boundary conditions that can be chosen for $\ell=0$ at $r \rightarrow 0$ consistently with self-adjoint extensions.

Let us parametrize the Robin boundary conditions by $\gamma$ and let us define the auxiliary quantity $\gamma_{\ell}$ by

$$
\gamma_{\ell}:= \begin{cases}\gamma \in[0, \pi), & \text { if } \ell=0, \\ 0, & \text { if } \ell>0 .\end{cases}
$$

The most general solution that is square integrable at the endpoint $r \rightarrow 0$ and yields self-adjoint extensions for the radial part of the Klein-Gordon operator can be written as

$$
R_{\gamma_{\ell}}(p r):=\cos \left(\gamma_{\ell}\right) R_{1}(p r)-\sin \left(\gamma_{\ell}\right) R_{2}(p r) .
$$

Since $R_{1}$ is the principal solution, the self-adjoint extension determined by taking $\gamma=0$ corresponds to the Friedrichs extension and we refer to this particular case as the Dirichlet boundary condition. Also, note that for $\ell>0$, the solution (13) indeed reduces to the principal solution.

By standard methods of singular Sturm-Liouville theory ([17], Chap. 10) we can construct the Green's function of the radial equation (7). Following exactly the same procedure as in [12-15] and invoking precisely the same symmetry arguments for performing the contour integration, the spectral resolution of the radial Green's function gives rise to the following identity [mind that $p=p\left(\omega^{2}\right)$ as per Eq. (8)]:

$$
\int_{m_{0}^{2}}^{\infty} d \omega^{2} \frac{p}{\pi} \frac{R_{\gamma_{\ell}}(p r) R_{\gamma_{\ell}}\left(p r^{\prime}\right)}{\cos \left(\gamma_{\ell}\right)^{2}+p^{2} \sin \left(\gamma_{\ell}\right)^{2}}=-\frac{\delta\left(r-r^{\prime}\right)}{q(r)} .
$$

The identity above fixes the integral kernel of the two-point functions, as we show in the next section.

\section{Ground and thermal states}

A physically sensible two-point function on a global monopole spacetime $\mathcal{M}$ is a positive bidistribution $\mathcal{G}_{\beta, \gamma} \in \mathcal{D}^{\prime}(\mathcal{M} \times \mathcal{M})$ that solves the Klein-Gordon equation in each entry and is of local Hadamard form. Taking into account that $\mathcal{M}$ is static and spherically symmetric, and given the addition formula for the spherical harmonics, we consider the following ansatz for the integral kernel of $\mathcal{G}_{\beta, \gamma}$ :

$$
\mathcal{G}_{\beta, \gamma}\left(x, x^{\prime}\right)=\sum_{\ell=0}^{\infty} \int_{0}^{\infty} d \omega \mathcal{T}_{\beta}\left(t, t^{\prime}\right) \mathcal{R}_{\gamma}\left(r, r^{\prime}\right) \Xi\left(\theta, \varphi, \theta^{\prime}, \varphi^{\prime}\right),
$$


where $x=(t, r, \theta, \varphi) \in \mathcal{M}$,

$$
\begin{aligned}
\Xi\left(\theta, \varphi, \theta^{\prime}, \varphi^{\prime}\right):= & \frac{2 \ell+1}{4 \pi} P_{\ell}\left(\cos (\theta) \cos \left(\theta^{\prime}\right)\right. \\
& \left.+\sin (\theta) \sin \left(\theta^{\prime}\right) \cos \left(\varphi-\varphi^{\prime}\right)\right),
\end{aligned}
$$

and $P_{\ell}$ is the Legendre function of first kind.

The time function $\mathcal{T}_{\beta}\left(t, t^{\prime}\right)$ specifies the support of $\mathcal{G}_{\beta, \gamma}$ with respect to the Fourier frequency $\omega$. In turn, its support specifies the nature of the corresponding state. The twopoint function of a ground state has support over positive $\omega$ frequencies; hence, we take

$$
\mathcal{T}_{\infty}\left(t, t^{\prime}\right)=e^{-i \omega\left(t-t^{\prime}-i 0^{+}\right)} .
$$

The two-point function of a thermal state at inverse temperature $\beta$ with respect to the Killing field $\partial_{t}$ is one that satisfies the KMS condition; see, e.g., Ref. [18]. This property is guaranteed to hold when taking

$$
\mathcal{T}_{\beta}\left(t, t^{\prime}\right)=\frac{e^{-i \omega\left(t-t^{\prime}-i 0^{+}\right)}}{1-e^{-\beta \omega}}+\frac{e^{+i \omega\left(t-t^{\prime}+i 0^{+}\right)}}{e^{\beta \omega}-1} .
$$

Note that $\mathcal{T}_{\infty}\left(t, t^{\prime}\right)$ is in fact the zero-temperature limit $(\beta \rightarrow \infty)$ of $\mathcal{T}_{\beta}\left(t, t^{\prime}\right)$ and that the forms of both the time and the angular parts of ansatz (15) are restricted by the symmetries of the spacetime. However, the radial part $\mathcal{R}_{\gamma}\left(r, r^{\prime}\right)$ depends on the particular form of the metric. Specifically, it is related to the Green's function of the radial part of the Klein-Gordon equation and it is uniquely determined, up to the choice of boundary conditions, by the canonical commutation relations, as we state in the following.

Analogously to the cases detailed in [12-15], it happens that ansatz (15) satisfies the canonical commutation relations provided the function $\mathcal{R}_{\gamma}$ is symmetric under the mapping $r \leftrightarrow r^{\prime}$ and if

$$
\int_{0}^{\infty} d \omega^{2} \mathcal{R}_{\gamma}\left(r, r^{\prime}\right)=-\frac{\delta\left(r-r^{\prime}\right)}{\eta(r)},
$$

where $\eta(r)$ is such that $\eta(r) \sin (\theta)=\sqrt{|g|}=\alpha^{2} r^{2} \sin \theta$. On the other hand, expression (19) is closely related to the spectral resolution of the Green's function of the radial equation. By comparing expressions (14) with (19), we directly obtain

$\mathcal{R}_{\gamma}\left(r, r^{\prime}\right)=\Theta\left(\omega-m_{0}\right) \frac{p}{\pi \alpha^{2}} \frac{R_{\gamma_{\ell}}(p r) R_{\gamma_{\ell}}\left(p r^{\prime}\right)}{\cos \left(\gamma_{\ell}\right)^{2}+p^{2} \sin \left(\gamma_{\ell}\right)^{2}}$.

Two-point functions constructed as above are guaranteed to yield physically sensible dynamics due to Wald and Ishibashi's work concerning static nonglobally hyperbolic spacetimes [6] and to be of local Hadamard form due to a general result by Sahlmann and Verch regarding the UV behavior of ground and thermal states on static spacetimes [19]. Altogether, we conclude that two-point functions with integral kernels given by (15), (16), and (20) characterize well-defined thermal states at inverse-temperature $\beta \in$ $(0, \infty)$ with respect to the Killing field $\partial_{t}$ when $\mathcal{T}_{\beta}\left(t, t^{\prime}\right)$ is given by Eq. (18). For $\mathcal{T}_{\beta}\left(t, t^{\prime}\right)$ given instead by the limiting case (17), (15) then characterizes a ground state and we shall denote it $\mathcal{G}_{\infty, \gamma}$.

We emphasize that even though expression (15) seems rather abstract, the integral can be analytically performed in some particular cases; e.g., for $\alpha \rightarrow 1$ and $\gamma=0$ it gives the standard closed-form expressions on Minkowski spacetime. Still, for a general set of parameters, expression (15) is suitable for numerical analyses. In particular, when considering field fluctuations, numerical integration can be performed after taking the coincidence limit $x^{\prime} \rightarrow x$ by invoking the Lebesgue dominated convergence theorem.

\section{TRANSITION RATE}

Consider an Unruh-DeWitt detector with energy gap $\Omega$ following a static trajectory of fixed spatial coordinates $(r, \theta, \varphi)$. The Fourier transform of $\mathcal{G}_{\infty, \gamma}$, given by (15) with (17), along such trajectory gives the transition rate of the detector when coupled to the ground state for an infinite proper time, as per (4):

$$
\begin{aligned}
\dot{\mathcal{F}}_{\infty, \gamma}(r)= & \frac{\Theta\left(-\Omega-m_{0}\right) \sqrt{\Omega^{2}-m_{0}^{2}}}{2 \pi \alpha^{2}} \\
& \times \sum_{\ell=0}^{\infty} \frac{2 \ell+1}{\cos \left(\gamma_{\ell}\right)^{2}+\left(\Omega^{2}-m_{0}^{2}\right) \sin \left(\gamma_{\ell}\right)^{2}} \\
& \times\left[R_{\gamma_{\ell}}\left(\sqrt{\Omega^{2}-m_{0}^{2}} r\right)\right]^{2} .
\end{aligned}
$$

Note that $\dot{\mathcal{F}}_{\infty, \gamma}(r)$ is independent of the angular position of the detector and vanishes identically for excitations $(\Omega>0)$, as expected to occur for a Boulware-like ground state. When coupled to a thermal state, as per (15) with (18), the transition rate reads instead

$$
\dot{\mathcal{F}}_{\beta, \gamma}(r)=\frac{\operatorname{sign}(\Omega)}{e^{\beta \Omega}-1}\left[\left.\dot{\mathcal{F}}_{\infty}(r)\right|_{\Omega \mapsto-|\Omega|}\right] .
$$

Both expressions (21) and (22) hold for massive, arbitrarily coupled fields and $\alpha \in(0,1]$. The $\ell$ sum can be analytically performed only in particular limiting cases. Yet, in any case, numerical analysis are easily performed due to the fast convergence of the sum in $\ell$. In the following, we discuss the transition rate of a detector coupled to a thermal state for a massless, minimally or conformally coupled, scalar field, and we study its behavior with respect to its distance from the naked singularity. 


\section{A. Minimal coupling}

Before discussing the numerical analysis, let us present some analytical considerations. For now, set $\xi=0$. In the limit $\alpha \rightarrow 1$, the spacetime corresponds simply to Minkowski spacetime with a boundary ${ }^{1}$ at $r \rightarrow 0$. Let us consider this scenario for the sake of comparison, denoting

$$
\dot{\mathcal{F}}_{\beta, \gamma}^{\operatorname{Mink}}(r):=\lim _{\alpha \rightarrow 1} \dot{\mathcal{F}}_{\beta, \gamma}(r) .
$$

In addition, for $\gamma=0$ (23) yields the expected result on Minkowski spacetime with no boundary:

$$
\dot{\mathcal{F}}_{\beta, 0}^{\mathrm{Mink}}=\frac{\Theta\left(|\Omega|-m_{0}\right)}{2 \pi} \frac{\operatorname{sign}(\Omega) \sqrt{\Omega^{2}-m_{0}^{2}}}{e^{\beta \Omega}-1} .
$$

Given that $\lim _{r \rightarrow 0} j_{\nu}(p r)=\delta_{\nu, 0}$ and that $\nu=0$ only if $\ell=0$ (and $\xi=0$ ), it follows that at $r \rightarrow 0$ only the $\ell=0$ mode contributes to $\dot{\mathcal{F}}_{\beta, \gamma}(\Omega)$. To put it in another way, in the $r \rightarrow 0$ limit the detector is only affected by $s$ waves. This is expected since we recover spherical symmetry as we approach the singularity. Consequently, for any $\gamma \geq 0$, close to the naked singularity we have

$$
\lim _{r \rightarrow 0} \dot{\mathcal{F}}_{\beta, \gamma}^{\text {Mink }}(r)=\dot{\mathcal{F}}_{\beta, 0}^{\text {Mink }} \cdot c_{\gamma}
$$

where $c_{\gamma}$ is a constant given by

$$
c_{\gamma}:=\left.\lim _{r \rightarrow 0} \frac{R_{\gamma_{\ell}}(p r)^{2}}{\cos \left(\gamma_{\ell}\right)^{2}+\left(\Omega^{2}-m_{0}^{2}\right) \sin \left(\gamma_{\ell}\right)^{2}}\right|_{\ell=0} .
$$

For arbitrary $\alpha$ instead, the following holds [see Eq. (21)]:

$$
\lim _{r \rightarrow 0} \dot{\mathcal{F}}_{\beta, \gamma}(r)=\frac{1}{\alpha^{2}} \lim _{r \rightarrow 0} \dot{\mathcal{F}}_{\beta, \gamma}^{\mathrm{Mink}}(r) .
$$

The behavior of the transition rate as the detector approaches the naked singularity on a global monopole, as given by (27), is analogous to that of a detector approaching a cosmic string. The Unruh-deWitt detector around a cosmic string was studied in Ref. [20] considering the Dirichlet boundary condition. There, the same behavior for the transition rate was obtained, namely,

$$
\lim _{r \rightarrow 0} \dot{\mathcal{F}}_{\beta, 0}(r)=\frac{1}{\alpha^{2}} \lim _{r \rightarrow 0} \dot{\mathcal{F}}_{\beta, 0}^{\text {Mink }} .
$$

Notice that Eq. (27) gives a more general result since it does not depend on the choice of the boundary condition. We should emphasize that the admissible boundary conditions at the global monopole singularity $r \rightarrow 0$ are much simpler

\footnotetext{
${ }^{1}$ Precisely, $r \rightarrow 0$ is actually the line $(t, 0, \theta, \varphi)$ with topology $\mathbb{R} \times S^{2}$, which is not a mathematical boundary but can be seen as a physical boundary.
}

than the ones at the cosmic string singularity $z=0$ (see [21], for instance). This justifies our choice for the global monopole as a toy model for spacetimes with naked singularities.

In addition, for all $r$, the contribution from the $\ell=0$ mode is such that

$$
\left.\dot{\mathcal{F}}_{\beta, \gamma}(r)\right|_{\ell=0}=\left.\frac{1}{\alpha^{2}} \dot{\mathcal{F}}_{\beta, \gamma}^{\operatorname{Mink}}(r)\right|_{\ell=0}
$$

Explicitly, for $m_{0}=0$, the transition (22) simplifies to

$\dot{\mathcal{F}}_{\beta, \gamma}(r)=\frac{1}{2 \pi \alpha^{2}} \frac{\Omega}{e^{\beta \Omega}-1} \sum_{\ell=0}^{\infty} \frac{(2 \ell+1)\left[R_{\gamma_{\ell}}(|\Omega| r)\right]^{2}}{\cos \left(\gamma_{\ell}\right)^{2}+\Omega^{2} \sin \left(\gamma_{\ell}\right)^{2}}$.

Expression (29) with $\xi=0$ is the one we consider in the following numerical analysis. For that, we perform the sum in $\ell$ from zero up to $\ell_{\max }$, as specified in the captions of each plot.

\section{With respect to $\alpha$}

Let us consider the Dirichlet case $\gamma=0$. As illustrated in Fig. 1, at large $r, \dot{\mathcal{F}}_{\beta, \gamma}(r)$ approximates, and oscillates around, the value of the transition rate in Minkowski, i.e.,

$$
\lim _{r \rightarrow \infty} \dot{\mathcal{F}}_{\beta, \gamma}(r) \sim \dot{\mathcal{F}}_{\beta, \gamma}^{\text {Mink }}(r) .
$$

The behavior close to the singularity is most clear in Fig. 2, which is consistent with Eq. (27). Note that as $r \rightarrow 0$, all curves converge to the same value as that for $\alpha=1$.

\section{With respect to $\gamma$}

Figure 3 encapsulates the main result we find. Namely, for any boundary condition $\gamma>0$, the transition rate diverges at $r \rightarrow 0$. Explicitly, using Eqs. (23)-(27), it

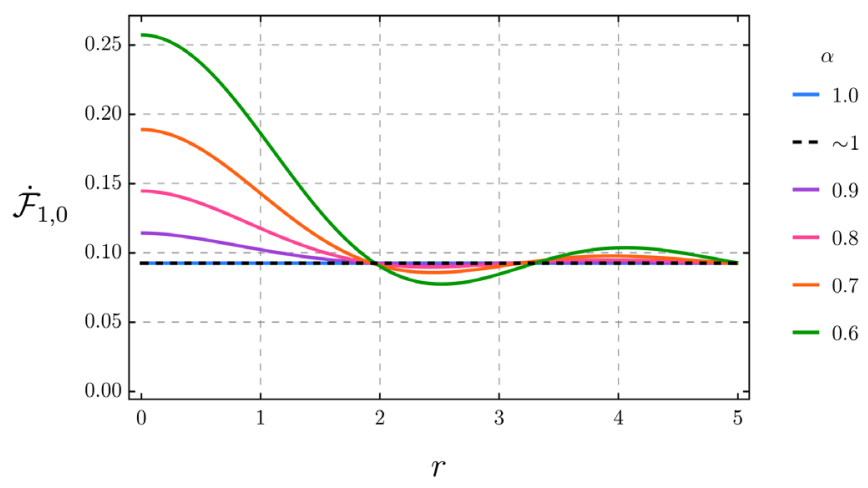

FIG. 1. The transition rate for the thermal state with $m_{0}=0$, $\xi=0, \beta=1, \Omega=1, \gamma=0, \ell_{\max }=10$ and, from top to bottom with respect to the apex, $\alpha \in\{0.6,0.7,0.8,0.9,0.99999,1.0\}$. Note that the dashed line, for which $\alpha$ is close to 1 , shows essentially the same behavior as for $\alpha=1$. 


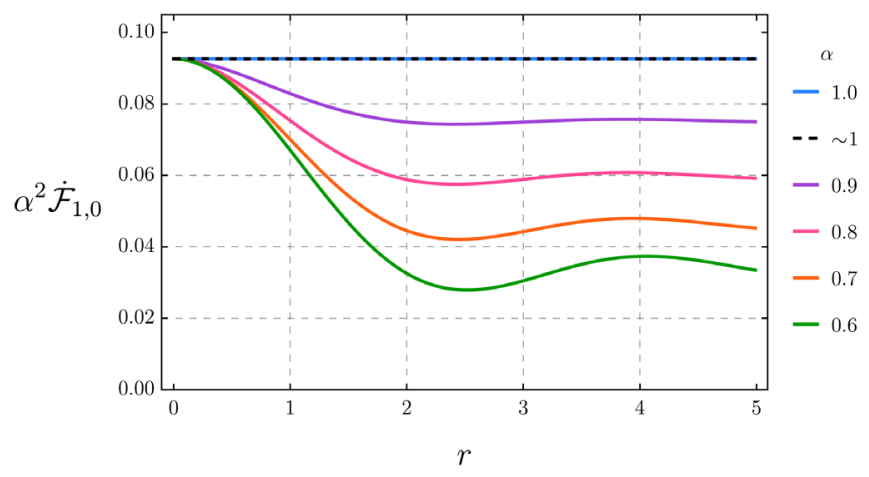

FIG. 2. The transition rate multiplied by $\alpha^{2}$ with the same parameters of Fig. 1.

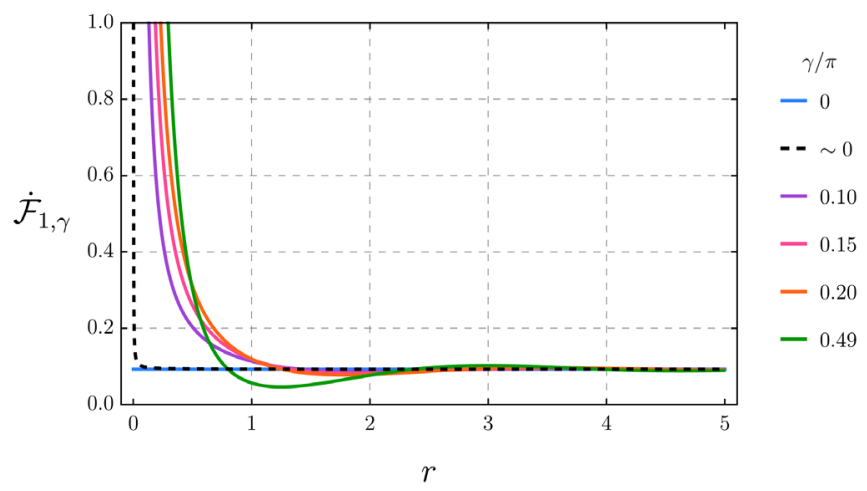

FIG. 3. The transition rate for the thermal state with $m_{0}=0$, $\xi=0, \beta=1, \Omega=1, \alpha=0.99999, \ell_{\max }=10$ and several values of $\gamma$. The dashed line corresponds to $\gamma / \pi=0.001$ and it shows a drastically different behavior as that of $\gamma=0$.

follows that close to the singularity the transition rate diverges with $r^{-2}$ if $\gamma \neq 0$ since

$$
\dot{\mathcal{F}}_{\beta, \gamma}(r) \stackrel{r \rightarrow 0}{\sim} \sigma_{\beta, \gamma}\left[\cos (\gamma)+\frac{\sin (\gamma)}{r}\right]^{2},
$$

where

$$
\sigma_{\beta, \gamma}:=\frac{1}{\alpha^{2}} \frac{\dot{\mathcal{F}}_{\beta, 0}^{\text {Mink }}}{\cos (\gamma)^{2}+\Omega^{2} \sin (\gamma)^{2}}>0
$$

In other words, only for the Dirichlet boundary condition does the spontaneous emission rate of a detector interacting with a thermal state remain finite at the naked singularity.

In Sec. IV A, we show that the quantum fluctuations as well as the energy supplied by the field are also divergent when the transition rate is divergent.

\section{B. Conformal coupling}

For $\xi=\frac{1}{6}, \alpha \in(0,1)$, and $\gamma \geq 0$, the parameter $\nu$ is never zero. This implies that in the limit $r \rightarrow 0$ even the $\ell=0$

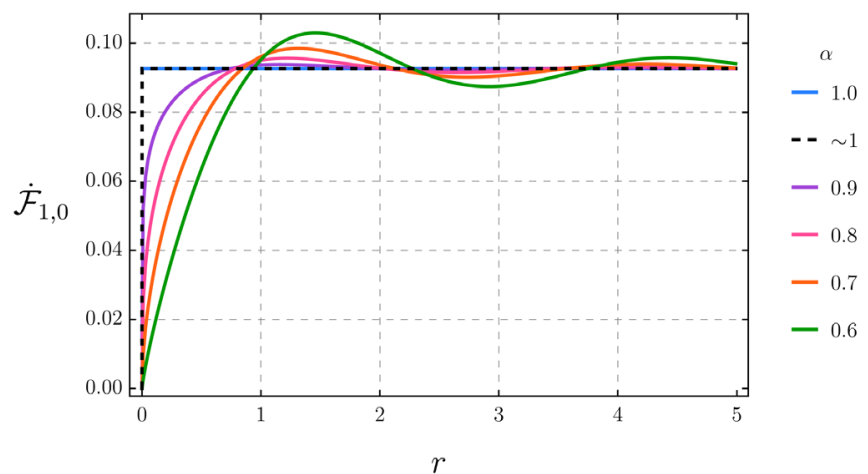

FIG. 4. The transition rate for the thermal state with $m_{0}=0$, $\xi=\frac{1}{6}, \beta=1, \Omega=1, \gamma=0, \ell_{\max }=10$ and several $\alpha$ 's.

contribution vanishes for $\gamma=0$; hence, the transition rate, given by Eq. (29) for a massless field, satisfies

$$
\lim _{r \rightarrow 0} \dot{\mathcal{F}}_{\beta, 0}(r)=0
$$

It follows that, as a function of $r$ for several $\alpha$ 's, the behavior of the transition rate illustrated in Fig. 4 is quite different from the minimally coupled case as in Fig. 1. However, for large $r, \dot{\mathcal{F}}_{\beta, \gamma}(r)$ also approximates, oscillating around, its respective value on Minkowski spacetime. The plot of $\dot{\mathcal{F}}_{\beta, \gamma}(r)$ for several boundary conditions is analogous to Fig. 3 in the sense that, for $\gamma>0$, the transition rate diverges at the singularity, with the only difference being that in this case it vanishes in the limit $r \rightarrow 0$ for $\gamma=0$.

Taking into account the asymptotic behavior of the Bessel functions ([16], Chap. 10), Eqs. (21) and (22) give

$$
\dot{\mathcal{F}}_{\beta, \gamma}(r)^{r \rightarrow 0} \tilde{\sigma}_{\beta, \gamma} \frac{\sin ^{2}(\gamma)}{r^{2\left(1+\nu_{0}\right)}},
$$

where

$$
\tilde{\sigma}_{\beta, \gamma}:=\sigma_{\beta, \gamma} \frac{2^{2 \nu_{0}} \Gamma\left(\nu_{0}+\frac{1}{2}\right)^{2}}{\pi \Omega^{2 \nu_{0}}}>0,
$$

with $\sigma_{\beta, \gamma}$ given by Eq. (32) and

$$
\nu_{0}:=\left.\nu\right|_{\ell=0}=\frac{-1+\sqrt{1+\frac{4\left(1-\alpha^{2}\right)}{3 \alpha^{2}}}}{2}>0 .
$$

\section{DISCUSSION}

Given that excitations cannot occur for a static detector coupled to a ground state, the thermal contribution solely accounts for the spontaneous emission displayed by a detector coupled to a thermal state. To understand the behavior of the detector in the latter case, as summarized in the last section, we analyzed the thermal contribution to the 
ground-state fluctuations and to the energy density. Before discussing the results, let us properly define these two quantities.

Let $\Delta \mathcal{G}_{\beta, \gamma}\left(x, x^{\prime}\right):=\mathcal{G}_{\beta, \gamma}\left(x, x^{\prime}\right)-\mathcal{G}_{\infty, \gamma}\left(x, x^{\prime}\right)$, where the two-point functions are given by (15), respectively, with (18) and (17). The thermal contribution to the ground-state fluctuations is given by

$$
\Delta \mathcal{G}_{\beta, \gamma}(r):=\lim _{x^{\prime} \rightarrow x}\left\{\Delta \mathcal{G}_{\beta, \gamma}\left(x, x^{\prime}\right)\right\} .
$$

Note that the coincidence limit above does not depend on the time and angular coordinates.

The energy-momentum tensor for the thermal state renormalized with respect to the ground state is defined as (see $[22,23]$ for details on Hadamard renormalization)

$$
\left\langle: T_{\mu \nu}(r):\right\rangle_{\beta, \gamma}=\lim _{x^{\prime} \rightarrow x}\left\{\mathcal{D}_{\mu \nu}\left(x, x^{\prime}\right)\left[\Delta \mathcal{G}_{\beta, \gamma}\left(x, x^{\prime}\right)\right]\right\},
$$

where the differential operator $\mathcal{D}_{\mu \nu}\left(x, x^{\prime}\right)$ is given by

$$
\begin{aligned}
\mathcal{D}_{\mu \nu}\left(x, x^{\prime}\right):= & (1-2 \xi) g_{\nu}^{\nu^{\prime}}\left(x, x^{\prime}\right) \nabla_{\mu} \nabla_{\nu^{\prime}}-2 \xi \nabla_{\mu} \nabla_{\nu}+G_{\mu \nu} \\
& +g_{\mu \nu}\left[2 \xi \square+\left(2 \xi-\frac{1}{2}\right) g_{\rho}{ }^{\rho^{\prime}}\left(x, x^{\prime}\right) \nabla^{\rho} \nabla_{\rho^{\prime}}-\frac{1}{2} m_{0}^{2}\right] .
\end{aligned}
$$

Accordingly, the energy density of the renormalized thermal state is simply the time-time component:

$$
E_{\beta, \gamma}(r):=\left\langle: T_{00}(r):\right\rangle_{\beta, \gamma} .
$$

For convenience, we omit the explicit expression for $E_{\beta, \gamma}(r)$, which is quite extensive and can be found in [24].

In the following sections, we summarize the results for the minimally and conformally coupled cases. What we find is that the transition rate, the thermal fluctuations, and the energy density are intertwined. For the minimally coupled case, as we approach the naked singularity, these three quantities diverge for $\gamma>0$, and are finite for $\gamma=0$. What is more, for $\ell=0$, the three quantities contrast with their counterparts in Minkowski spacetime in the same manner [as in Eq. (28)], viz.

$\{$ quantity for general $\alpha\}=\frac{1}{\alpha^{2}}\{$ quantity for $\alpha=1\}$.

Since the detector only sees the $\ell=0$ mode in the limit $r \rightarrow 0$, when $\xi=0$, relation (41) holds true when we consider such "quantity" to be the transition rate itself (summed up to $\infty$ ) or the thermal fluctuations. For the conformally coupled case, however, the association between the three quantities of interest is rather intricate and their relations with their counterparts on Minkowski spacetime do not respect Eq. (41). Most interesting is the fact that in the conformally coupled case, the energy density diverges at the singularity even when we set the Dirichlet boundary condition.

\section{A. Minimal coupling}

For $m_{0}=0$ and $\xi=0$, we have

$$
\begin{aligned}
\Delta \mathcal{G}_{\beta, \gamma}(r)= & \sum_{\ell=0}^{\infty} \int_{0}^{\infty} d \omega \frac{(2 \ell+1)}{2 \pi^{2} \alpha^{2}} \frac{\omega}{e^{\beta \omega}-1} \\
& \times \frac{\left[R_{\gamma_{\ell}}(\omega r)\right]^{2}}{\cos \left(\gamma_{\ell}\right)^{2}+\omega^{2} \sin \left(\gamma_{\ell}\right)^{2}} .
\end{aligned}
$$

Defining $\Delta \mathcal{G}_{\beta, \gamma}^{\mathrm{Mink}}$ as $\Delta \mathcal{G}_{\beta, \gamma}(r)$ with $\alpha \rightarrow 1$, we find that

$$
\lim _{r \rightarrow 0} \Delta \mathcal{G}_{\beta, \gamma}(r)=\frac{1}{\alpha^{2}} \lim _{r \rightarrow 0} \Delta \mathcal{G}_{\beta, \gamma}^{\operatorname{Mink}}(r),
$$

and

$$
\left.\Delta \mathcal{G}_{\beta, \gamma}(r)\right|_{\ell=0}=\left.\frac{1}{\alpha^{2}} \Delta \mathcal{G}_{\beta, \gamma}^{\mathrm{Mink}}(r)\right|_{\ell=0} .
$$

That is, as we approach the singularity, the behavior of the thermal fluctuations, given by (43), is analogous to that of

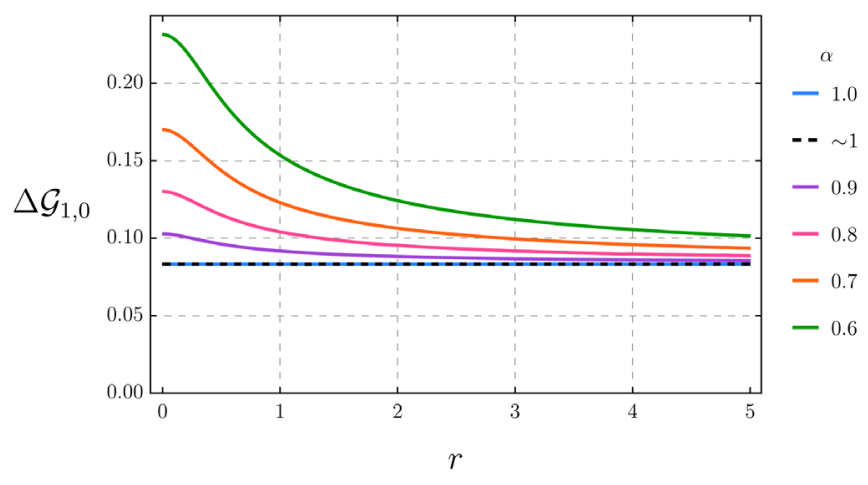

FIG. 5. Thermal fluctuations for $m_{0}=0, \xi=0, \beta=1, \gamma=0$, $\ell_{\max }=50$ and, from top to bottom with respect to the apex, $\alpha \in\{0.6,0.7,0.8,0.9,0.99999,1.0\}$. Note that the behavior for $\alpha=0.99999$ (dashed line) and for $\alpha=1$ are indistinguishable.

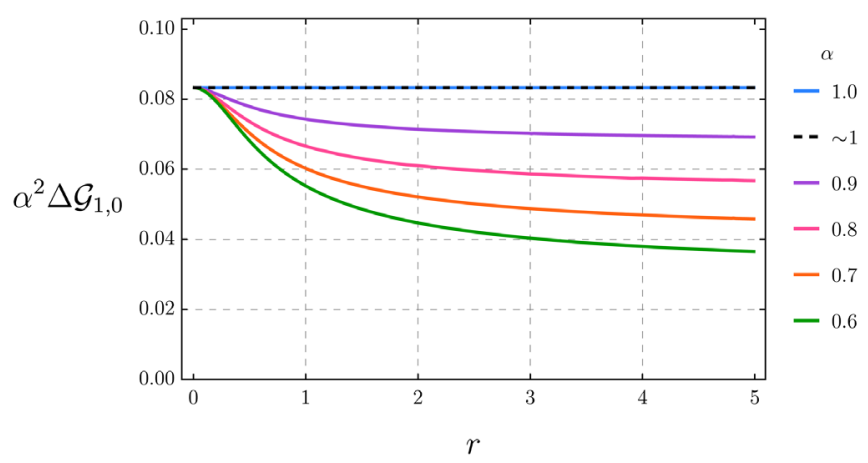

FIG. 6. Thermal fluctuations multiplied by $\alpha^{2}$ with the same parameters as in Fig. 5. 


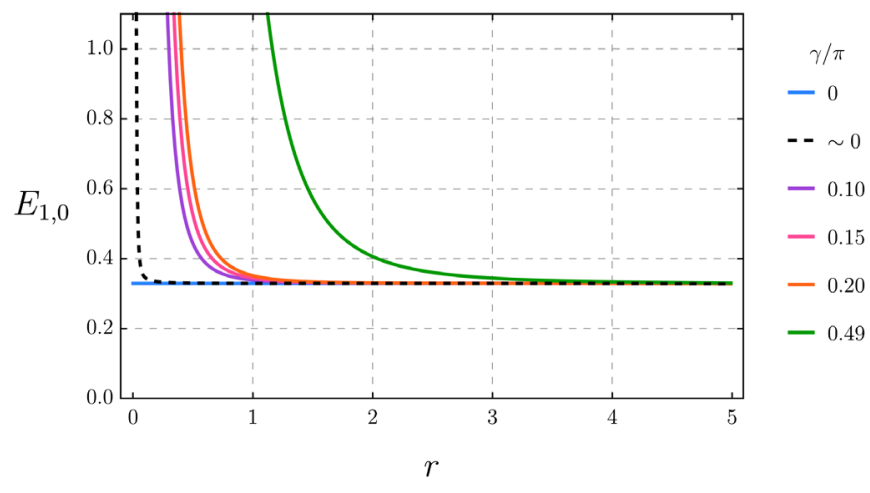

FIG. 7. Energy density for $m_{0}=0, \xi=0, \beta=1, \alpha=0.99999$, $\ell_{\max }=50$, and several values of $\gamma$. The dashed line corresponds to $\gamma / \pi=0.001$.

the transition rate, given by (27). This behavior is illustrated by Figs. 2 and 6, respectively. The same holds for the $\ell=0$ mode, given expressions (28) and (44). With respect to the boundary condition, the thermal fluctuations behave analogously to the transition rate, as in Fig. 6. That is, as $r \rightarrow 0$, $\Delta \mathcal{G}_{\beta, \gamma}(r)$ is finite if and only if $\gamma=0$.

Regarding $E_{\beta, \gamma}(r)$ with $m_{0}=0$ and $\xi=0$, first and foremost, we find that the energy density converges at $r \rightarrow 0$ only for the Dirichlet boundary condition, as shown in Fig. 7. The divergence of the energy at the naked singularity is consistent with the divergence of the spontaneous emission rate of the detector illustrated in Fig. 6. In addition, by performing numerical fits we obtained that the leading behavior of the energy density as we approach the singularity is given by

$$
E_{\beta, \gamma}(r) \stackrel{r \rightarrow 0}{\sim} \begin{cases}\rho_{\beta}, & \text { if } \gamma=0, \\ \frac{\tilde{\beta}_{\beta, \gamma}}{r^{4}}, & \text { if } \gamma>0,\end{cases}
$$

where $\rho_{\beta}$ and $\tilde{\rho}_{\beta, \gamma}$ are nonspecified constants with respect to $r$. In Fig. 8, we show the behavior of $\tilde{\rho}_{\beta, \gamma}$ with respect to the boundary condition for several values of $\alpha$. Moreover, as it

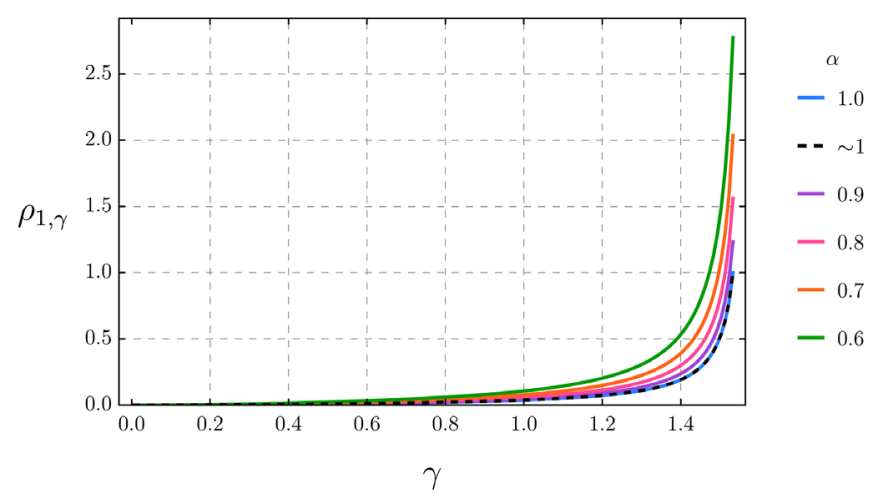

FIG. 8. The parameter $\rho_{\beta, \gamma}$ as a function of $\gamma$ for the thermal state with $m_{0}=0, \xi=0, \beta=1, \ell_{\max }=10$ and, from top to bottom with respect to the apex, $\alpha \in\{0.6,0.7,0.8,0.9,0.99999,1.0\}$. happens for the transition rate and for the thermal fluctuations, it holds that

$$
\left.E_{\beta, \gamma}(r)\right|_{\ell=0}=\left.\frac{1}{\alpha^{2}} E_{\beta, \gamma}^{\mathrm{Mink}}(r)\right|_{\ell=0} .
$$

\section{B. Conformal coupling}

For the same reason that the transition rate vanishes in the conformally coupled scenario, the thermal fluctuations (37) also vanish in the limit $r \rightarrow 0$. What is most interesting is that the energy density actually diverges at the naked singularity-even for the Dirichlet boundary conditionand yet, the transition rate vanishes there. For convenience, we illustrate the behavior of the thermal fluctuations, in Fig. 9, and of the energy density, in Fig. 10, considering only the $\ell=0$ mode.

For $\xi=\frac{1}{6}$, since numerical convergence is not as direct as in the minimally -coupled case, we performed a numerical fit only for the most relevant case of $\alpha=0.99999$ (with grand unified theories in mind); we obtained that the leading behavior of the energy density as we approach the singularity is

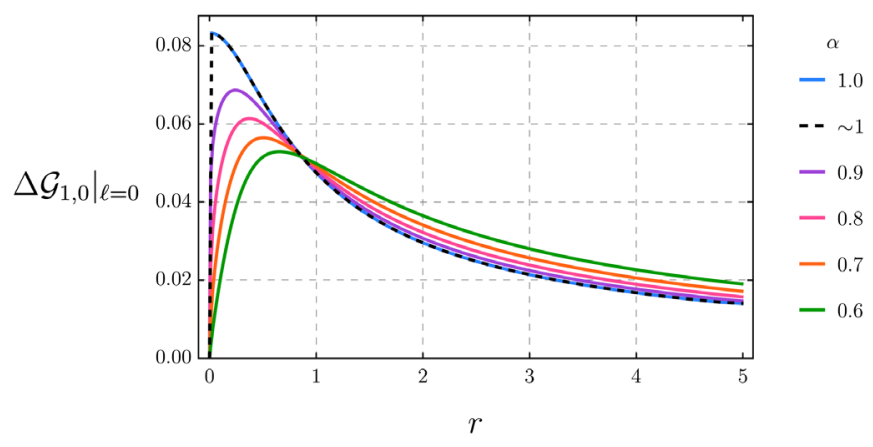

FIG. 9. Thermal fluctuations for $m_{0}=0, \xi=\frac{1}{6}, \beta=1, \gamma=0$, $\ell_{\max }=0$ and several $\alpha$ 's.

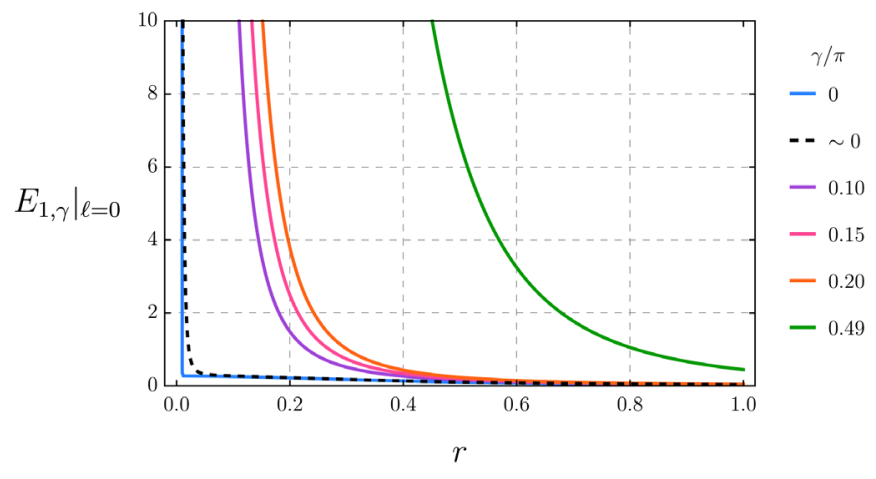

FIG. 10. Energy density for $m_{0}=0, \quad \xi=\frac{1}{6}, \quad \beta=1$, $\alpha=0.99999, \ell_{\max }=0$ and several $\gamma$ 's. 


$$
E_{\beta, \gamma}(r) \stackrel{r \rightarrow 0}{\sim} \begin{cases}\frac{\rho_{\beta}}{r^{2}}, & \text { if } \gamma=0, \\ \frac{\tilde{\rho}_{\beta, \gamma}}{r^{4\left(\nu_{0}+1\right)}}, & \text { if } \gamma>0,\end{cases}
$$

where $\rho_{\beta, \gamma}$ and $\tilde{\rho}_{\beta, \gamma}$ are positive constants with respect to $r$ [not necessarily equal to those of Eq. (45)], while $\nu_{0}$ is defined in Eq. (36). However, we do expect that for other values of $\alpha, E_{\beta, \gamma}(r)$ behaves similarly to Eq. (47) with a subexponential divergence rate.

\section{CONCLUSION}

The propagation of quantum fields on nonglobally hyperbolic spacetimes is not, in general, uniquely determined by the initial data on a spacelike surface. Wald and Ishibashi tackled this problem in Refs. [5,6], where they prescribed a way of extracting sensible dynamics for such fields by finding positive self-adjoint extensions of the spatial component of the differential wave operator. These self-adjoint extensions, in turn, are prescribed by appropriate boundary conditions at the boundaries of the spacetime. Any quantity extracted from the quantum fields depends crucially on the choice of the boundary condition.

In this paper we investigated how the boundary condition at the classical singularity $r \rightarrow 0$ of the global monopole spacetime affects the transition rate as measured by a particle detector. These (Robin) boundary conditions turn out to be extremely simple to handle, which makes the global monopole spacetime a very attractive toy model in the study of quantum effects due to naked singularities.

We considered a static Unruh-deWitt detector at a distance $r$ from the singularity. For a massless, minimally coupled scalar fields on its ground state, the rate of excitation is zero in the infinite time interaction limit. This is expected for inertial observers on a general static spacetime. However, when the global monopole is immersed on a thermal bath with temperature $T \sim 1 / \beta$, the induced extra thermal fluctuations creates a nontrivial scenario for the excitation of the detector. These thermal fluctuations are finite at the singularity $r \rightarrow 0$ only for the Dirichlet boundary condition $(\gamma=0)$ and diverge for any other Robin boundary condition $(\gamma>0)$. The transition rate behaves similarly in this limit. Nevertheless, the expected Minkowski thermal fluctuations are recovered in the $r \rightarrow \infty$ limit regardless of the choice of the boundary condition. This, in turn, induces the usual Minkowski transition rate for the quantum field on a thermal state. Moreover, for any boundary condition parametrized by $\gamma \in[0, \pi)$, we have

$$
\frac{\dot{\mathcal{F}}_{\beta, \gamma}\left(0^{+}\right)}{\dot{\mathcal{F}}_{\beta, \gamma}^{\text {Mink }}\left(0^{+}\right)}=\frac{\Delta \mathcal{G}_{\beta, \gamma}\left(0^{+}\right)}{\Delta \mathcal{G}_{\beta, \gamma}^{\text {Mink }}\left(0^{+}\right)}=\frac{E_{\beta, \gamma}\left(0^{+}\right)}{E_{\beta, \gamma}^{\text {Mink }}\left(0^{+}\right)}=\frac{1}{\alpha^{2}},
$$

which shows that the transition rate, the thermal fluctuations and the energy density of the renormalized thermal state are amplified by the presence of the singularity in exactly the same way.

The situation is rather different for conformally coupled fields. Relation (41) does not hold, in general. Most importantly, the energy density diverges at $r \rightarrow 0$ for all $\gamma \geq 0$, and yet both the thermal fluctuations and the transition rate vanish. That is, even if there is an infinite amount of energy available, the detector will not undergo an excitation if the quantum field is not fluctuating.

Finally, in this work we showed that naked singularities manifest thermal effects with a nontrivial behavior with respect to the admissible boundary conditions in a static scenario. The divergence of the quantities mentioned indicates that strong backreaction effects may arise, hence an appealing research avenue to follow would be to study them, particularly, to see if such effects would clothe the singularity or not. In addition, in a future work it would be most interesting to study a dynamical scenario, either considering an accelerated detector or a model of collapse, in order to approach the question of whether naked singularities can evaporate a bit more accurately.

\section{ACKNOWLEDGMENTS}

The work of L. S. C, who is grateful for discussions with C. Dappiaggi, was supported by a Ph.D. scholarship from the University of Pavia. We also thank the referee for the useful comments and suggestions given.
[1] J. M. M. Senovilla, Singularity theorems in general relativity: Achievements and open questions, Einstein Stud. 12, 305 (2012).

[2] A. Vilenkin and E. P. S. Shellard, Cosmic Strings and Other Topological Defects, Cambridge Monographs on Mathematical Physics (Cambridge University Press, Cambridge, England, 2000).
[3] S. W. Hawking, Black hole explosions?, Nature (London) 248, 30 (1974).

[4] M. Casals, A. Fabbri, C. Martínez, and J. Zanelli, Quantum dress for a naked singularity, Phys. Lett. B 760, 244 (2016).

[5] R. M. Wald, Dynamics in nonglobally hyperbolic, static space-times, J. Math. Phys. (N.Y.) 21, 2802 (1980). 
[6] A. Ishibashi and R. M. Wald, Dynamics in nongloballyhyperbolic static spacetimes: II. General analysis of prescriptions for dynamics, Classical Quant. Grav. 20, 3815 (2003).

[7] J. P. M. Pitelli and P. S. Letelier, Quantum singularities around a global monopole, Phys. Rev. D 80, 104035 (2009).

[8] V.S. Barroso and J. P. M. Pitelli, Vacuum fluctuations and boundary conditions in a global monopole, Phys. Rev. D 98, 065009 (2018).

[9] F. D. Mazzitelli and C. O. Lousto, Vacuum polarization effects in global monopole space-times, Phys. Rev. D 43, 468 (1991).

[10] F. C. Carvalho and E. R. Bezerra de Mello, Vacuum polarization for a massless scalar field in the global monopole space-time at finite temperature, Classical Quant. Grav. 18, 1637 (2001).

[11] J. Louko and A. Satz, Transition rate of the Unruh-DeWitt detector in curved spacetime, Classical Quant. Grav. 25, 055012 (2008).

[12] C. Dappiaggi and H. Ferreira, Hadamard states for a scalar field in anti-de Sitter spacetime with arbitrary boundary conditions, Phys. Rev. D 94, 125016 (2016).

[13] C. Dappiaggi, H. Ferreira, and A. Marta, Ground states of a Klein-Gordon field with Robin boundary conditions in global anti-de Sitter spacetime, Phys. Rev. D 98, 025005 (2018).

[14] F. Bussola, C. Dappiaggi, H. R. C. Ferreira, and I. Khavkine, Ground state for a massive scalar field in the BTZ spacetime with Robin boundary conditions, Phys. Rev. D 96, 105016 (2017).
[15] L. S. Campos and C. Dappiaggi, Ground and thermal states for the Klein-Gordon field on a massless hyperbolic black hole with applications to the anti-Hawking effect, Phys. Rev. D 103, 025021 (2021).

[16] NIST Digital Library of Mathematical Functions, https:// dlmf.nist.gov/10, Accessed: 2021-09-29.

[17] A. Zettl, Sturm-Liouville Theory (American Mathematical Society, Providence, 2005).

[18] O. Bratteli and D. W. Robinson, Operator Algebras and Quantum Statistical Mechanics. Vol. 2: Equilibrium States. Models in Quantum Statistical Mechanics, Texts and Monographs in Physics Vol. 2 (Springer, New York, 2012).

[19] H. Sahlmann and R. Verch, Passivity and microlocal spectrum condition, Commun. Math. Phys. 214, 705 (2000).

[20] P. C. W. Davies and V. Sahni, Quantum gravitational effects near cosmic strings, Classical Quant. Grav. 5, 1 (1988).

[21] B.S. Kay and U.M. Studer, Boundary conditions for quantum mechanics on cones and fields around cosmic strings, Commun. Math. Phys. 139, 103 (1991).

[22] Y. Decanini and A. Folacci, Hadamard renormalization of the stress-energy tensor for a quantized scalar field in a general spacetime of arbitrary dimension, Phys. Rev. D 78, 044025 (2008).

[23] T. P. Hack and V. Moretti, On the stress-energy tensor of quantum fields in curved spacetimes - comparison of different regularization schemes and symmetry of the Hadamard/ Seeley-DeWitt coefficients, J. Phys. A 45, 374019 (2012).

[24] L.S. Campos, Mathematica notebook with the numerical analyses of this paper, https://github.com/lissadesouzacampos/ thermal_effects_on_a_global_monopole.git. 